\title{
Phagocytosis in Plant Protoplasts
}

\author{
Katsumi Ueda, Kyoko Tan, Fumihiko Sato* and Yasuyuki Yamada* \\ Biological Laboratory, Nara Women's University, Nara 630 and *Department \\ of Agricultural Chemistry, Kyoto University, Kyoto 606
}

\begin{abstract}
Protoplasts from mesophyll cells from leaves of Nicotiana tabacum and cultured cells of Rauwolfia serpentina were used. When polyethylene glycol was added to a mixture of these protoplasts, some protoplasts phagocytotically ingested another whole protoplast into their vacuoles. Phagocytosis occurred not only between protoplasts of the same species but also between those of different species. At the initial contact of two protoplasts, the cytoplasm between the plasma membrane and the tonoplast of the ingesting protoplast migrated from the area of contact and an invagination developed containing the other protoplast which was finally incorporated into the vacuole by closure of the invagination.
\end{abstract}

Phagocytosis, the ingestion by cells of fairly large granules or solid fragments into the cytoplasm, has been commonly observed in animal cells, especially in protozoa (7). In plants, cells are enclosed by cell walls which obstruct the passage of solid granules. It is natural, therefore, that phagocytosis has not been reported in plant cells.

Recently, methods of preparing plant protoplasts, which lack cell walls, have been developed $(4,15)$, and the ingestion by protoplasts of small particles such as polystyrene latex particles $(10,13)$, tobacco mosaic virus $(3,14)$, and the larger chloroplasts $(2,6)$ and nucleus $(11)$ has been reported. We have found that a plant protoplast is also able to incorporate another protoplast into its vacuole, as described below.

\section{MATERIALS AND METHODS}

Mesophyll cells from leaves of Nicotiana tabacum and suspension-cultured cells of Rauwolfia serpentina were used as the sources of the protoplasts as previously reported (16). The mesophyll cells and the cultured cells were separately treated with an enzyme solution containing 5\% cellulase (Onozuka R-10, Kinkiyakult), $1 \%$ macerozyme (Kinkiyakult), and $0.5 \mathrm{M}$ mannitol for $2 \mathrm{~h}$ at $35^{\circ} \mathrm{C}$, then they were filtered through $100 \mu \mathrm{m}$ steel mesh to remove cell clumps. Protoplasts which passed through the mesh were centrifuged and collected. The two kinds of protoplasts were then mixed, and polyethylene glycol (MW 7000 ) was added to the mixture to a final concentration of $20 \%$.

Protoplasts were fixed for electron microscopy $30 \mathrm{~min}, 2 \mathrm{~h}$, and $4 \mathrm{~h}$ after the addition of polyethylene glycol, with $5 \%$ glutaraldehyde buffered with phosphate at $\mathrm{pH} 7.4$ containing $0.4 \mathrm{M}$ mannitol. Protoplasts were treated for $30 \mathrm{~min}$ in the fixative, washed with phosphate buffer and post-fixed with $1 \%$ buffered osmium tetroxide for $2 \mathrm{~h}$. They were then washed with water, dehydrated with acetone, and embedded in Epon. Ultrathin sections were stained with lead citrate and examined under an electron microscope, HU 12A type. 


\section{RESULTS}

Immediately after the addition of polyethylene glycol, protoplasts began to fuse with one another or to ingest other protoplasts. The process of ingestion, by which one protoplast engulfs another, was recorded cinematographically under a light microscope. Figs. 1, 2, 3 and 4 are taken from a cinefilm, and show two protoplasts 3, 10, 20 and 40 min after coming into contact with each other. The smaller protoplast, which contains chloroplasts, originated from a mesophyll cell of Nicotiana tabacum and the larger one from Rauwolfia serpentina. These figures show the steps by which the Rauwolfia protoplast took up the tobacco protoplast. The tobacco protoplast is first engulfed by the Rauwolfia protoplast and is finally incorporated completely. The Rauwolfia protoplasts were able to take up either tobacco protoplasts or in other cases protoplasts of the same species. At the time of initial contact it could not be predicted by light microscopic observation which of the two protoplasts would be invaginated. Of course, in a complete uptake the larger of the two invaginated, but in some cases the smaller protoplast invaginated and became attached to the larger one like a cap.

Several stages of the uptake process were observed with an electron microscope. Fig. 5 shows a Rauwolfia protoplast contained in an invagination in a tobacco protoplast. In the invaginating protoplast no detectable layer of the cytoplasm was present beneath the plasma membrane of the invagination and the tonoplast closely lay beneath the plasma membrane, while in the engulfed protoplast a cytoplasm of normal appearance was present beneath the area of contact. Migration of the cytoplasm from the area of contact occurred just after adhesion in one of the protoplasts, and was the first sign of invagination and ingestion into the vacuole. In the case of fusion of two or more protoplasts, as shown in Fig. 6, the cytoplasm was thick beneath the two con-

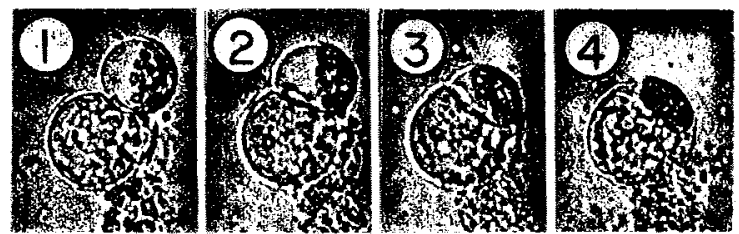

Figs. 1-4. Ingestion by a Rauwolfia protoplast of a tobacco protoplast, 3, 10, 20, and 40 min after contact. $\times 250$.

Fig. 5. Rauwolfia protoplast in an invagination in a tobacco protoplast. $\times 1,000$.

Fig. 6. Four protoplasts in the process of fusion. $\times 1,000$.

Fig. 7. Fusion of the outer envelope of a chloroplast of tobacco and the tonoplast of Rauwolfia at the point indicated by arrow B. The plasma membrane of Rauwolfia and the plasma membrane of tobacco also fuse with the outer envelope of the chloroplast of tobacco. $\times 66,000$. Arrows: See text.

Fig. 8. Fusion of the tonoplast and the outer envelope of a chloroplast at the points indicated by arrows. $\times 40,000$.

Fig. 9. Small protoplast in an invagination in a large protoplast. $\times 2,500$.

Fig. 10. Tobacco protoplast ingested in the vacuole of Rauwolfia. $\times 10,000$.

Fig. 11. Tobacco protoplast ingested in the vacuole of another tobacco protoplast. Arrows indicate droplets of polyethylene glycol. $\times 1,400$.

Fig. 12. A part of Fig. 11 enlarged. The plasma membrane of the ingested protoplast is intact. Droplets of polyethylene glycol (arrows) are seen in the chloroplasts and in the ground plasm of the cytoplasm. $\times 15,000$. 

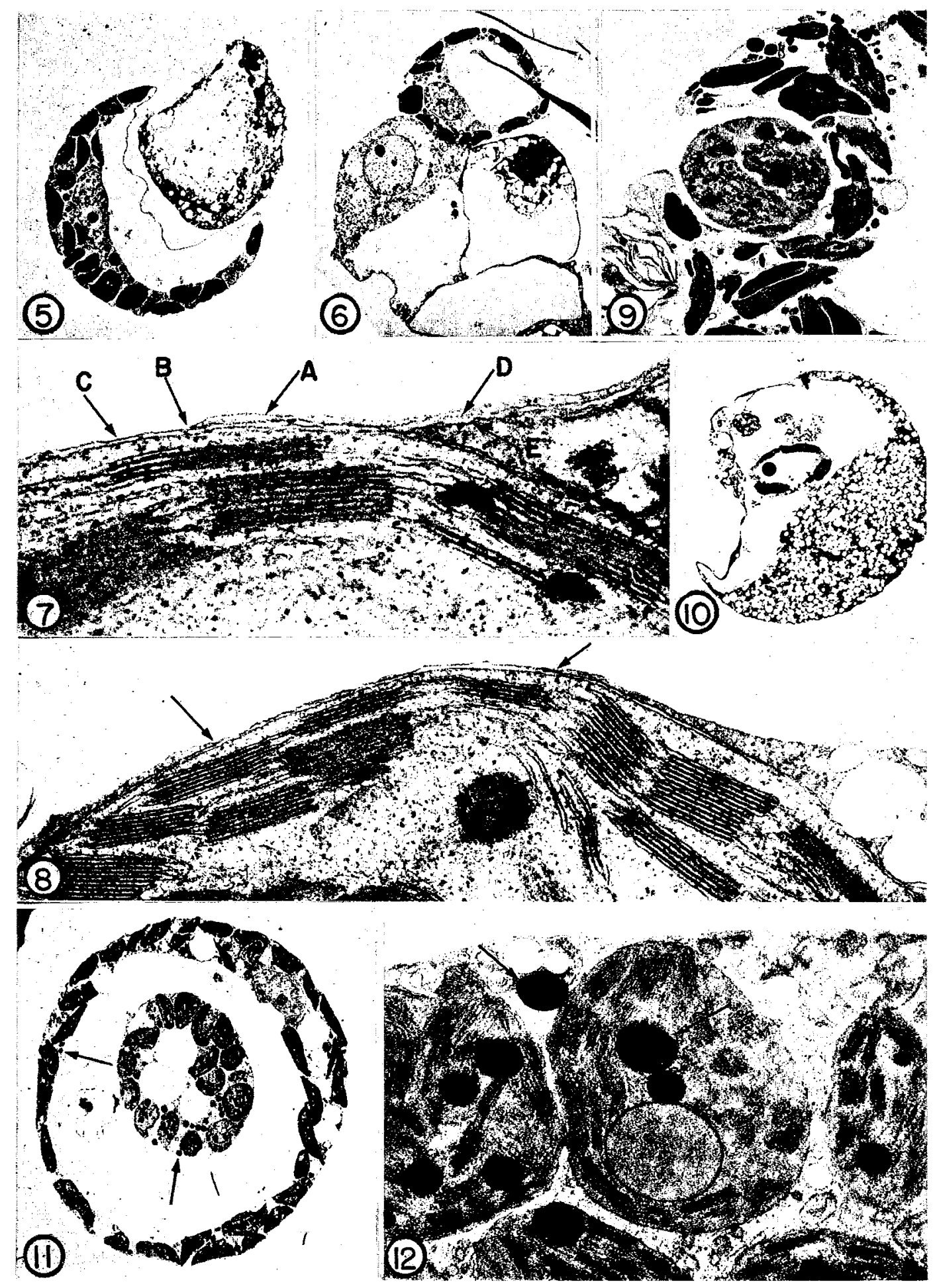
tacting plasma membranes or thin beneath both. Fusion between protoplasts, details of which will be published elsewhere, results in the disappearance of the two contacting plasma membranes and in the mixing of the cytoplasmic components.

Fusion of different kinds of cytoplasmic membranes was frequently observed in the uptake of protoplasts under the present experimental conditions. In Fig. 7, arrow C indicates the double membrane of a chloroplast envelope of a tobacco protoplast, arrow A the tonoplast membrane of Rauwolfia, and arrow B the point of fusion of the terminal of the tonoplast membrane with the outer membrane of the chloroplast envelope. Arrow D indicates the plasma membrane of the Rauwolfia protoplast and arrow $\mathrm{E}$ the plasma membrane of the tobacco protoplast. The plasma membranes lie in close proximity with their termini on the outer membrane of the chloroplast envelope. Fig. 8 also shows the fusion of the tonoplast membrane with the outer membrane of the chloroplast envelope at the point indicated by the arrows.

When the ingesting protoplast was much larger than the engulfed protoplast, and the cytoplasm was thick enough to enclose the ingested protoplast, the cytoplasm of the invaginating protoplast did not migrate from the contact region between the protoplasts. Fig. 9 shows the invagination of a large tobacco protoplast containing a smaller one. This invagination closely resembles that seen in normal phagocytosis.

Four hours after the addition of polyethylene glycol, many protoplasts containing another protoplast in the vacuole were observed. Some contained the same kind of protoplast, as shown in Fig. 11 (a tobacco protoplast in a tobacco protoplast), and some the other kind of protoplast, as shown in Fig. 10 (a tobacco protoplast in a Rauwolfia protoplast). The protoplasts in Figs. 10 and 11 were confirmed by light microscopy before sectioning to contain a protoplast completely enclosed in the vacuole. The enclosed tobacco protoplast in Fig. 11 has a plasma membrane, tonoplast, and normal cytoplasm. There is no continuous membrane surrounding the embedded protoplast between the tonoplast of the enclosing protoplast and the plasma membrane of the embedded protoplast, which confirms that the figure shows a protoplast enclosed in the vacuole rather than a sectional view of an invaginating protoplast. A part of the enclosed protoplast in Fig. 11 is enlarged in Fig. 12, which clearly reveals the normal appearance of the plasma membrane. Many electron dense droplets (indicated by arrows in Figs. 11 and 12), which may be polyethylene glycol, were observed in the groundplasm of the cytoplasm and in the chloroplasts. They could not be seen in the nuclei or the mitochondria.

\section{DISCUSSION}

Plants are usually autotrophic and, unlike animals, need not ingest organic substances. This fact does not mean that plant cells are less active in the uptake of organic and high molecular substances. If these substances can pass through the cell wall, they will be incorporated into the cytoplasm usually by pinocytosis (1). Not only organic substances but also ions are assumed to be pinocytotically incorporated into the cytoplasm of plant cells (5). Considering the occurrence of pinocytosis in plant cells enclosed by cell walls, the pinocytotic or phagocytotic incorporation of small particles into naked plant protoplasts as reported by some investigators $(3,10,13,14)$ is not unexpected.

Surprisingly, however, we have found that a protoplast ingested another whole protoplast into its vacuole. The process of ingestion started with the adhesion of two 
protoplasts followed by the formation of an invagination in the surface of one protoplast, which was seemingly similar to that found in pinocytosis and the phagocytosis of solutions or relatively small particles in animal cells. In the uptake of large particles seen in the amoeba, cell surface does not form invaginations, but protrudes and engulfs the particle (8). The difference between the surface protrusion and invagination may reflect the nature of the cells, that is, one type must actively catch and ingest particles for maintenance of life, and the other needs not. The plant cells from which the protoplasts are derived have cell walls, and they can not and need not take up large bodies. The phagocytotic phenomenon seen in the protoplasts may result from the increased activity of ingestion through the invaginations induced by polyethylene glycol. Polyethylene glycol was used by Kao and Michayluk (9) for the fusion of different kinds of protoplasts. They assumed that polyethylene glycol acts as a molecular bridge between the surfaces of adjacent protoplasts to initiate adhesion between them. Because the protoplasts treated with polyethylene glycol had many droplets in the cytoplasm (Figs. 11 and 12), polyethylene glycol may act to increase the endocytotic activity of the protoplasts. The increase in this activity to the extent that a whole protoplast is ingested would account for the phagocytotic phenomenon observed in the present investigation.

The characteristic feature of the phagocytosis of plant protoplasts is the direct uptake of a large body into the vacuole when the diameter of the body exceeds the thickness of the cytoplasm. The plasma membrane and the tonoplast of the invaginating protoplast lie in close proximity with no structured cytoplasm between them, and behave as a tight double membrane during the process of phagocytosis. The invagination in the surface of the protoplast leads directly into the vacuole, so that a protoplast enclosed by the invaginated membrane enters the vacuole after closure of the invagination.

Fusion between the plasma membrane and the outer envelope of the chloroplast or between the tonoplast and the outer envelope of the chloroplast was observed. All of these membranes consist of a bimolecular leaflet of lipids with proteins inserted into the lipid layer (12). The characteristics of the membranes of cell organelles seems to depend on the specific constituent proteins in the membranes, and not on the lipids. If two different membranes come into close contact and the specific proteins of both membranes might move away from the area of contact, leaving a homogeneous area mostly containing lipids, fusion of the two membranes may occur, because the structure of the lipid leaflet does not vary widely between membranes of different kinds. In the course of the ingestion of the protoplast, organelles and various cytoplasmic membranes are possibly subjected to unusually strong forces, which is suggested by the fact that the cytoplasm is removed from a wide area between the plasma membrane and the tonoplast. Fusion between different kinds of membranes may be derived from these unusual forces.

\section{REFERENCES}

1. Baker, D.A. and J.L. Hall. Pinocytosis, ATP-ase and ion uptake by plant cells. New Phytol. 72, 1281-1292, 1973

2. Bennetr, H.T. and T. ERIKSSON. Transfer of algal chloroplasts into protoplasts of higher plants. Planta (Berl.) 120, 71-79, 1974

3. Cocking, E.C. An electron microscopic study of the infection of isolated tomato fruit protoplast by tobacco mosaic virus. J. Gen. Virol. 4, 313-320, 1969 
4. Cocking, E.C. Plant cell protoplasts. -Isolation and development. Ann. Rev. Plant Physiol. 23, 29-50, 1972

5. Costerton, J.W.F. and E.A.C. MACrobBie. Ultrastructure of Nitella translucens in relation to ion transport. J. Exp. Bot. 21, 535-542, 1970

6. Davey, M.R., E. Frearson and J.B. Power. Polyethylene glycol-induced transplantation of chloroplasts into protoplasts: An ultrastructural assessment. Plant Sci. Lett. 7, 7-16, 1976

7. Jeon, K.W. The biology of Amoeba. Academic Press, New York. 1973

8. JEON, K.W. and M.S. JEON. Scanning electron microscope observation of Amoeba proteus during phagocytosis. J. Protozool. 23, 83-86, 1976

9. KAO, K.N. and M.R. Michayluk. A method for high-frequency intergeneric fusion of plant protoplasts. Planta (Berl.) 115, 355-367, 1974

10. MAYO, M.A. and E.C. CockING. Pinocytotic uptake of polystyrene latex particles by isolated tomato fruit protoplasts. Protoplasma 68, 223-230, 1969

11. Potrykus, I. and F. Hoffmann. Transplantation of nuclei into protoplasts of higher plants. Z. Pflanzenphysiol. 69, 287-289, 1973

12. SINGER, S.J. The molecular organization of biological membranes. Structure and function of biological membranes. ed. Rothrield Ll., Academic Press, New York. 1971

13. Suzuki, M., I. Takebe, S. KajtTa, Y. Honda and C. Matsui. Endocytosis of polystyrene spheres by tobacco leaf protoplasts. Exp. Cell Res. 105, 127-135, 1977

14. TAKEBE, I. and Y. OtsUKI. Infection of tobacco mesophyll protoplasts by tobacco mosaic virus. Proc. Nat. Acad. Sci. 64, 843-850, 1969

15. TAKEBE, I., Y. OTSUKI and S. AoKI. Isolation of tobacco mesophyll cells in intact and active states. Plant Cell Physiol. 9, 115-124, 1968

16. Yamada, Y. and M. NAKAminami. The isolation and fusion of protoplasts from alkaloid plant cells cultured in vitro. In Protoplastes et fusion de cellules somatique Vegetales, Centre National de la Recherche Agronomique, Paris. pp. 373-384, 1973

(Received for publication, September 22, 1977) 